\title{
Incidence and treatment of MR-ONJ in a cohort of oncological patients.
}

\author{
Giacomo Setti ${ }^{1}$, Giovanna Garuti ${ }^{2}$, Francesco Diamante ${ }^{2}$, Ugo Consolo $^{2}$, Pierantonio Bellini $^{2}$ \\ 1 Università degli Studi di Modena e Reggio Emilia \\ 2 Università degli Studi di Modena e Reggio Emilia
}

Funding: The author(s) received no specific funding for this work.

Potential competing interests: The author(s) declared that no potential competing interests exist.

\section{Abstract}

Antiresorptive and antiangiogenic therapy, as well as target therapy agents (such as drugs for immunotherapy, immunomodulators, enzyme or protein inhibitors), are widely administered in several life-threatening oncological and hematological diseases. Medication-related osteonecrosis of jaws (MR-ONJ) is an adverse effect which incidence is strongly related to primary disease history, therapy management and duration, systemic co-morbidities treatments, dental and oral health control.

A previously reported case series described a 10-year (2007-2016) single-center raw incidence of 47 MR-ONJ cases in a cohort of 1663 patients treated with antiresorptive drugs (2.8\%). The significant positive effect of a dental and oral health prevention protocol was appreciated by splitting the cohort into two timeframes, 2007-2010 and 2011-2016. During the first period, dental evaluation and treatment were not implemented and MR-ONJ incidence was $4.9 \%$; in the second one, the incidence dropped (1.9\%), suggesting the value of the applied protocol.

Between February 2020 and February 2021, 184 onco-hematological new patients were referred for comprehensive dental, oral health evaluation and treatment. Including subjects already in follow-up, overall 313 patients were in charge. In 12 months, MR-ONJ occurred in 5 cases (incidence 1.6\%). Altogether, 11 MR-ONJ cases were treated in the considered timeframe, of which 6 were previously diagnosed.

Following staging, treatment options ranged from conservative to surgical therapy, depending on extension, drug history, primary disease prognosis, and patient feasibility.

\section{Background:}

Antiresorptive and antiangiogenic therapy, as well as target therapy agents (such as drugs for immunotherapy, immunomodulators, enzyme or protein inhibitors), are widely administered in several life-threatening oncological and hematological diseases. Medication-related osteonecrosis of jaws (MR-ONJ) is an adverse effect whose incidence is strongly related to primary disease history, therapy management and duration, systemic comorbidities treatments, dental and oral health control.

A previously reported case series described a 10-year (2007-2016) single-center raw incidence of 47 MR-ONJ cases in a cohort of 1663 patients treated with antiresorptive drugs (2.8\%). The significant positive effect of a dental and oral health 
prevention protocol was appreciated by splitting the cohort into two timeframes, 2007-2010 and 2011-2016. During the first period, dental evaluation and treatment were not implemented and MR-ONJ incidence was $4.9 \%$; in the second one, the incidence dropped (1.9\%), suggesting the value of the applied protocol [1].

\section{Materials and Methods:}

A dedicated service for oncological patients (CLID Project) was activated by the Oncological Center and the Unit of Dentistry and Oral-Maxillofacial Surgery in 2010 [1]. Since the beginning, dentists, oral surgeons and dental hygienists have cooperated for diagnosis, support and treatment of oral and dental issues of such patients. The first visit, recalls and therapy appointments were scheduled by dental and cancer specialists together. Orthopantomogram evaluation, periodontal probing, dental and soft tissue examination were performed during the first visit, as well as oral professional hygiene. If patients were denture carriers, the prosthesis was evaluated, paying attention to local pressure points. When dental extraction or non-surgical periodontal therapy was planned, antiresorptive agent administration was delayed until successful healing of post extractive sockets and periodontal index improvement. Further examination and oral hygiene were scheduled for 4 months recall.

A yearly retrospective evaluation of referred patients for onco-hematological diseases was performed. All patients were treated with antiresorptive medications (denosumab alone as a first-choice treatment or secondarily to previous bisphosphonates therapy) after a comprehensive oral and dental evaluation.

Patients that had shown bone new exposure were considered for conservative or surgical therapy. Moreover, data regarding the outcome of MR-ONJ treatment that occurred in the considered timeframe were reported.

Selection between treatment options was tailored for the specific case. The first step was to evaluate whether the patient was eligible for surgical therapy. Patients with extremely compromised medical status or that could not suspend antiresorptive therapy due to oncological issues were deemed ineligible for surgical therapy and were considered for conservative treatment. As a second step, we evaluated the oral status of the patient: the presence of bone exposure and its extension, presence of chronic infection, pain, swelling or other conditions possibly affecting the patient's quality of life. Subjects eligible for surgery underwent a strict preoperative preparation: patients received professional oral hygiene 1 week before surgery and were invited to rinse with $0,2 \%$ chlorhexidine gluconate twice a day. LLLT was administered twice for 4 minutes at 0,6W in CW (diode laser, 975nm, Dentsply Sirona) on the exposed bone and its peripheral inflamed soft tissue and on surrounding healthy mucosa.

The surgical approach consisted of a full-thickness flap followed by sequestrectomy, bone debridement and recontouring [2]. To obtain flap mobility and passivation, periosteal incisions were carried out; multiple relieving incisions were performed only for small-sized defects. To ensure tension-free wound closure, continuous and simple interrupted sutures were applied [3]. The surgical area was then irradiated for LLLT. Post-operative instructions were given. Follow-up was scheduled at 3, 7, 10, 15, 21 days for LLLT repetition.

Applying the SIPMO protocol [4], surgery was performed under antibiotic prophylaxis: $1 \mathrm{~g}$ of Amoxicillin + Clavulanic Acid and $500 \mathrm{mg}$ of Metronidazole 3 times per day, starting six days before surgery if MRONJ was suppurating (stage $1 \mathrm{~b}$ or $2 \mathrm{~b}$ according to SIPMO classification) or starting two days before surgical treatment if suppuration or swelling were not present (stage 1a and 2a SIPMO). Same antibiotic therapy was carried on for 10 days after surgery or until the wound healing. 
Probiotic factors were administered to all patients for 1 month post antibiotics therapy to help gut flora restoring.

Patients with absolute or relative surgery contraindications received conservative treatment: $1 \mathrm{~g}$ of Amoxicillin + Clavulanic Acid and $500 \mathrm{mg}$ of Metronidazole 3 times per day, for 10 days in association to LLLT [5], performed every three days until the disappearance of swelling, pain and inflammation of the oral mucosa. In case of partial or incomplete remission, antibiotic therapy was repeated after 1 month as well as LLLT.

Follow-up appointments were scheduled at 1, 2, 3, 6, 9, 12 months after bone exposure treatment; all patients had a comprehensive dental and soft tissue examination, while professional oral hygiene was performed only if needed.

\section{Results:}

Between February 2020 and February 2021, 184 new onco-hematological patients were evaluated; 62 out of 184 patients were considered eligible for bone marrow transplantation; the remaining 122 patients were addressed to antiresorptive therapy due to bone metastatic spreading of lung, prostate or breast neoplasms. Overall, the patients addressed for followup were 313.

MR-ONJ occurred in 5 cases (incidence 1.6\%), which were all diagnosed for breast metastatic cancer (mean age 61,2; range between 52 and 70 years). Such new exposures were scored according to AAOMS staging system [6] as 2 stage 0 (40\%), 2 stage $1(40 \%)$ and 1 stage $2(20 \%)$. In addition, scoring based on SIPMO guidelines [4] was assigned as follows: 1 stage $1 \mathrm{a}(20 \%)$ and 4 stages $1 \mathrm{~b}(80 \%)$.

On the whole, 11 patients were treated for MR-ONJ following breast metastatic cancer therapy in the considered timeframe (mean age 66,4; range between 52 and 87 years). Among those, 6 had previously received bone exposure diagnosis (mean age 70,6; range between 54 and 87 years). The adverse drug reaction occurred in 8 patients $(72,7 \%)$ treated only with denosumab and in 3 cases $(27,3 \%)$ administered with denosumab and zoledronic acid for more than 24 months. The average denosumab administration (120 mg monthly) before the adverse drug reaction was 24 in patients who received denosumab alone and 35 in patients who previously received zoledronic acid.

MR-ONJ were clinically staged according to AAOMS [6]: 4 stage 0 (36,4\%), 3 stage 1 (27,2\%) and 4 stage $2(36,4 \%)$. Moreover, a comprehensive evaluation of tri-dimensional radiographic imaging allowed a further staging according to SIPMO [4]. Nine stage 1 ( $81,8 \%$, of which 7 stage $1 \mathrm{~b}$ and 2 stage $1 \mathrm{a})$ and 2 stage $2 \mathrm{~b}(18,2 \%)$ were identified. Also, cases classified as stage 0 according to AAOMS charting [6] were scored as $1 \mathrm{~b}$ according to the SIPMO staging - system [4]. Bone necrosis affected the lower jaw in 8 out of 11 cases $(72,7 \%)$, while in 3 cases $(27,3 \%)$ involved the maxilla. The outbreak of exposures was related to dentures sores (2 cases, $18,2 \%$ ) or followed dental extraction ( 2 cases, $18,2 \%)$; remaining manifestations involved the alveolar bone without previous odontogenic or periodontal infection (7 cases, $63,6 \%)$.

Eight patients $(72,7 \%)$ were treated with surgery and low-level laser therapy (LLLT) $[7,8]$. Two patients $(18,2 \%)$ were treated with both antibiotic therapy and LLLT $[5,8]$. One patient resulted not eligible for surgery and conservative treatment was planned; therefore, the severe worsening of her health condition led to demise before any treatment.

No patients had shown new signs of bone exposures, swelling, pain or mucosal alterations during follow-up.

\section{Conclusions :}

The incidence of MR-ONJ in the selected time frame was $1.6 \%$ (5 cases in overall 313 patients). Since the application of the CLID Project for onco-hematological patients, MR-ONJ incidence was about 1.9\% (period 2011-2016). Most of the 
exposures were early identified and the low stage at diagnosis likely represents a favorable clinical condition. The presented results were almost superimposable to our previous epidemiological report, strengthening the role of a strict recall program. Currently, with the limitation of a short post-treatment follow-up, complete clinical and radiographic healing was achieved in 10 patients; one patient had died before the administration of any specific therapy.

\section{References}

1. Badea M., Setti G., Panari L., Consolo U., Bellini P.. “[1]”. Min Stomatol 2018;67(3)suppl.1:9.

2. Rupel K., Ottaviani G., Gobbo M., Contardo L., Tirelli G., Vescovi P., et al. A systematic review of therapeutical approaches in bisphosphonates-related osteonecrosis of the jaw. Oral Oncol 2014;50(11):1049-57. doi: [2]

3. Stockmann P., Vairaktaris E., Wehrhan F., Seiss M., Schwarz S., Spriewald B., et al. ${ }^{[3]}$. Support Care Cancer 2010;18(4):449-60 doi: 10.1007/s00520-009-0688-1.

4. Campisi G., Bedogni A., Fusco V. "[4]” (Versione 2.0; Luglio 2020)

5. Romeo U., Galanakis A., Marias C., Del Vecchio A., Tenore G., Palaia G., Vescovi P., Polimeni A. Observation of Pain Control in Patients with Bisphosphonate-Induced Osteonecrosis Using Low-Level Laser Therapy: Preliminary Results. Photomed Laser Surg 2011;29(6):447-452. doi: ${ }^{[5]}$

6. Ruggiero SL., Dodson TB., Fantasia J., Goodday R., Aghaloo T., Mehrotra B., et al. American association of oral and maxillofacial surgeons position paper on medication-related osteonecrosis of the jaw - 2014 update. J Oral Maxillofac Surg 2014;72(10):1938-56. doi: [6].

7. Vescovi P., Manfredi M., Merigo E., Guidotti R., Meleti M., Pedrazzi G., et al. Early surgical laser-assisted management of bisphosphonate-related osteonecrosis of the jaws (BRONJ): a retrospective analysis of 101 treated sites with longterm follow-up. Photomed Laser Surg 2012;30(1):5-13. doi: [7].

8. Weber JBB., Camilotti RS., Ponte ME. Efficacy of laser therapy in the management of bisphosphonate-related osteonecrosis of the jaw (BRONJ): a systematic review. Lasers Med Sci 2016;31(6):1261-72. doi: 10.1007/s10103016-1929-4[8]

\section{References}

1. ' Badea M., Setti G., Panari L., Consolo U., Bellini P.. (2018). Retrospective analysis of BP therapy in 1663 oncological and hematological patients between 2007 and 2016. Effect of dental prevention on ONJ incidence. Minerva Stomatologica, vol. 67(3)suppl.1:9. .

2. 'Katia Rupel, Giulia Ottaviani, Margherita Gobbo, Luca Contardo, et al. (2014). A systematic review of therapeutical approaches in bisphosphonates-related osteonecrosis of the jaw (BRONJ). Oral Oncology, vol. 50 (11), 1049-1057. doi:10.1016/j.oraloncology.2014.08.016.

3. ^Philipp Stockmann, Eleftherios Vairaktaris, Falk Wehrhan, Martin Seiss, et al. (2009). Osteotomy and primary wound closure in bisphosphonate-associated osteonecrosis of the jaw: a prospective clinical study with 12 months follow-up. Support Care Cancer, vol. 18 (4), 449-460. doi:10.1007/s00520-009-0688-1.

4. ' Campisi G., Bedogni A., Fusco V.. (2020). Raccomandazioni clinico-terapeutiche sull'osteonecrosi delle ossa mascellari (ONJ) farmaco-relata e sua prevenzione. PalermoUniversityPess. 
5. 'Umberto Romeo, Alexandros Galanakis, Christos Marias, Alessandro Del Vecchio, et al. (2011). Observation of Pain Control in Patients with Bisphosphonate-Induced Osteonecrosis Using Low Level Laser Therapy: Preliminary Results. Photomedicine and Laser Surgery, vol. 29 (7), 447-452. doi:10.1089/pho.2010.2835.

6. 'Salvatore L. Ruggiero, Thomas B. Dodson, John Fantasia, Reginald Goodday, et al. (2014). American Association of Oral and Maxillofacial Surgeons Position Paper on Medication-Related Osteonecrosis of the Jaw-2014 Update. Journal of Oral and Maxillofacial Surgery, vol. 72 (10), 1938-1956. doi:10.1016/j.joms.2014.04.031.

7. ^Paolo Vescovi, Maddalena Manfredi, Elisabetta Merigo, Rebecca Guidotti, et al. (2012). Early Surgical Laser-Assisted Management of Bisphosphonate-Related Osteonecrosis of the Jaws (BRONJ): A Retrospective Analysis of 101 Treated Sites with Long-Term Follow-Up. Photomedicine and Laser Surgery, vol. 30 (1), 5-13. doi:10.1089/pho.2010.2955.

8. `João Batista Blessmann Weber, Renata Stifelman Camilotti, Monique Estér Ponte. (2016). Efficacy of laser therapy in the management of bisphosphonate-related osteonecrosis of the jaw (BRONJ): a systematic review. Lasers Med Sci, vol. 31 (6), 1261-1272. doi:10.1007/s10103-016-1929-4. 